\section{hommes}

\section{Hommes \& migrations}

Revue française de référence sur les dynamiques

migratoires

\section{8 | 2014}

Les Paris des migrants

\title{
Les dessous de la Ville-lumière
}

Fantasmes et nausée littéraires des étudiants africains à Paris

(1945-1975)

\section{Nicolas Treiber}

\section{(2) OpenEdition \\ Journals}

Édition électronique

URL : http://journals.openedition.org/hommesmigrations/3018

DOI : 10.4000/hommesmigrations.3018

ISSN : 2262-3353

Éditeur

Musée national de l'histoire de l'immigration

Édition imprimée

Date de publication : 1 octobre 2014

Pagination : 151-158

ISBN : 978-2-919040-29-2

ISSN : $1142-852 X$

Référence électronique

Nicolas Treiber, «Les dessous de la Ville-lumière », Hommes \& migrations [En ligne], 1308 | 2014, mis en ligne le 01 octobre 2017, consulté le 30 avril 2019. URL : http://journals.openedition.org/ hommesmigrations/3018; DOI : 10.4000/hommesmigrations.3018 


\section{LES DESSOUS \\ DE LA VILLE-LUMIĖRE \\ FANTASMES ET NAUSÉE LITTÉRAIRES DES ÉTUDIANTS AFRICAINS À PARIS (1945-1975)}

par NICOLAS TREIBER, doctorant en littérature, université d'Aix-Marseille-I.

Pour les étudiants africains qui l'ont arpentée depuis les années 1930, la Ville-lumière semble constellée de zones d'ombre. Paris, la capitale mondiale des arts et des lettres de l'après-guerre qui attire les artistes de tous horizons, est aussi celle d'un empire colonial. Aussi, dans certains espaces de la ville relève-t-on les marques de la colonisation. Des traces dont certains écrivains comme Ousmane Socé, Aké Loba, Cheikh Hamidou Kane ou Saïdou Bokoum nourrissent leur premier roman, dressant de Paris un portrait en clair-obscur.

"Ô toi qui voyages je te donne un conseil, prends-le aussitôt / Vois ce qui te convient avant de vendre et d'acheter", Ya Rayeh, Dahmane el Harrachi.

Paris est un pôle d'attraction symbolique qui sécrète sa propre mythologie. Si l'on en croit l'un de ses arpenteurs minutieux, Walter Benjamin, sa topographie même est un palimpseste de toutes les écritures qu'elle a su éveiller, concentrer, et qu'elle conserve dans la moindre de ses ruelles. "Paris est la grande salle de lecture d'une bibliothèque que traverse la Seine ${ }^{1}$." On imaginerait volontiers des rangées interminables de beaux-livres tout à la gloire de la capitale française dont le rayonnement international n'est plus à prouver. Mais l'entrée dans cette grande bibliothèque urbaine au tournant des indépendances, sous la conduite des auteurs africains, dévoile un autre parcours, une autre réalité : un apprentissage de la ville dont il s'agit d'interroger le fonctionnement au prisme de l'imaginaire colonial. Durant la période coloniale, Paris n'était qu'à quelques encablures des écoles de brousse. Avec la création de l'Union française en 1946, les étudiants africains colonisés sont de plus en plus nombreux à venir achever leur formation en France. Selon les estimations du ministère de l'Éducation, leur effec- 
tif passe de 250 en 1946 à 5500 en 1960². Parmi eux, de nombreux écrivains en herbe vont puiser dans leur parcours migratoire vers la capitale de la métropole coloniale la matière de leur premier roman. On se reportera notamment à Ousmane Socé, Mirages de Paris (1937) ; Aké Loba, Kocoumbo, l'étudiant noir (1960); Cheikh Hamidou Kane, L'Aventure ambiguë (1961) ; Camara Laye, Dramouss (1966) ; Yambo Ouologuem, Le Devoir
Avant même qu'ils en

aient foulé le sol, Paris est

déjà fortement présent

en Afrique, dans l'esprit

des élèves colonisés.

En tant que dernière étape

de la formation coloniale,

Paris joue un rôle crucial dans

l'entreprise de colonisation

culturelle de violence (1968) ; Saïdou Bokoum, Chaîne (1974) ${ }^{3}$. Ces textes se placent à hauteur d'homme, dans le regard et l'esprit du migrant qui découvre la ville, faisant souvent du Paris de ses rêves le terrain de ses cauchemars. Des années 1930 aux années 1970, ils ont en commun une peinture sombre de la vie parisienne dont les étudiants africains feraient l'expérience. Nous avons cherché à comprendre leur insistance, au-delà de la césure historique des indépendances africaines, à mettre en scène la déchéance de personnages en proie aux doutes quant à l'issue de leur aventure migratoire, à la tentation du suicide, au déclassement social.

Paris est le terrain privilégié de la rencontre entre la population française et les migrations (post) coloniales, dont la littérature africaine d'expression française n'a cessé de baliser les impasses, les échecs mais aussi les possibilités d'échanges, de dialogue, une fois réglés leurs comptes aux miroirs de l'exotisme pervers, au racisme de l'ignorance et à la suffisance d'une culture qui s'est construite sur l'arrogance de sa supériorité fantasmée. Il s'agit donc d'étudier la façon dont fonctionne le discours-objet ${ }^{4}$ de Paris chez les auteurs africains qui ont fréquenté la ville entre l'après-guerre et le début des années 1970. Car la mise en scène des représentations de la capitale française et des différents discours idéologiques qu'elle suscite semble épouser une certaine géographie de la ville. Un itinéraire qui conduit le migrant des mirages élyséens à ses zones d'ombre, ces espaces interlopes relégués à la marge de la grande parade urbaine.

\section{Dans les miroitements de la Ville-lumière}

Avant même qu'ils en aient foulé le sol, Paris est déjà fortement présent en Afrique, dans l'esprit des élèves colonisés. En tant que dernière étape de la formation coloniale, Paris joue un rôle crucial dans l'entreprise de colonisation culturelle. Ainsi, Kocoumbo, qui part en France en 1946, bénéficiant de l'alignement des programmes scolaires sur ceux de la métropole voulu par la récente Union française, est littéralement sous le charme de Paris : "Il n'y avait plus que Paris dans son coeur. (...) Le jeune homme arriva ainsi à se persuader... dans l'ensemble Paris s'apprêtait à le recevoir. (...) Se considérant déjà comme un étranger, il ne se mêlait plus ni aux uns ni aux autres (KO, p. 32)." C'est cependant une culture de papier glacé qui domine ses rêves, pour preuve : "la pile de catalogues des grands magasins de Paris qui lui servait d'oreiller (KO, p. 30)”.

Non content d'exclure des siens l'étudiant sur le départ, l'imaginaire de Paris est le produit d'une sous-culture colonisée sur laquelle, selon Bernard Mouralis, repose la diffusion culturelle du système colonial : "le choix, s'il existe, ne peut être qu'entre une caricature de culture européenne et ce que le colonisateur a laissé subsister de la culture autochtone ${ }^{5 "}$.

2. Pascal Blanchard, Alain Mabanckou, La France noire, Paris, La Découverte, 2011, p. 185.

3. Les références des citations des romans sur lesquels porte la présente étude seront placées dans le texte accompagnées des cigles entre parenthèses : Ousmane Socé, Mirages de Paris (MDP), Nouvelles éditions latines, 1937 ; Aké Loba, Kocoumbo, l'étudiant noir (KO), Flammarion, 1960 ; Cheikh Hamidou Kane, L’Aventure ambiguë (AA), Julliard, 1961 ; Camara Laye, Dramouss (DRA), Plon, 1966 ; Yambo Ouologuem, Le Devoir de violence (DV), Seuil, 1968 ; Saïdou Bokoum, Chaine (CH), Denoël, 1974. Précisons toutefois que les textes d'Ousmane Socé et de Camara Laye ne sont pas des premiers romans, les auteurs ayant respectivement publié auparavant : Karim. Roman Sénégalais (1935) et L'Enfant noir (1953). 4. Voir Michel Foucault, L'Archéologie du savoir, Paris, Gallimard, 1969, p. 190. Par la réécriture d'un “discours-objet”, Foucault désigne l'objectif d'une archéologie que nous voulons littéraire. Non pas exhumer les traces d'un temps révolu, mais mettre au jour les conditions de possibilités de discours qui travaillent notre présent. 5. Bernard Mouralis, Littérature et développement, Paris, Silex, 1984, p. 54. 
Les publicités venues de Paris tracent ainsi à gros trait les contours d'un territoire scintillant où les produits de la technologie dont on fait la réclame se mêlent au ballet idéal des figures de la capitale mondiale de la vie culturelle de l'époque.

Depuis les années 1920, la relation entre Paris et les Africains s'est tissée sur le double fond de l'engouement pour les "attractions" ethnographiques venues des colonies - en témoigne l'Exposition coloniale de 1931 dont rend compte Mirage de Paris d'Ousmane Socé - et du développement du racisme. En réponse, l'écriture de Paris s'est élaborée, chez ces auteurs africains, dans le creuset problématique de la fascination et du rejet.

\section{Une hétérotopie colonisante}

Pour les migrants, Paris est un espace autre qui a pénétré leur discours par le biais de représentations colonisées qui les attirent irrémédiablement. Mais la ville est aussi une gigantesque machine à fabriquer de l'autre. D'emblée, l'imaginaire de Paris distingue ceux qui s'y rendent, à l'instar de Kocoumbo, devant sa malle, sur le bateau qui le conduit vers Marseille: "Sur le dessus de celle-ci sétalait en gros caractères OUDJO KOCOUMBO. Il se pencha pour ajouter à côté : étudiant. Un scrupule le fit hésiter et il se mit à consulter un petit dictionnaire. Il se décida enfin à tracer d'une plume ferme : étudiant à Paris (KO, p. 63)." Durant la période coloniale, le voyage vers Paris est vécu, par tout son cercle social, comme une onction pour celui qui a la chance de partir. Cette sacralité renvoie à l'usage que fait Michel Foucault du concept d'hétérotopie $^{6}$. L'hétérotopie désigne la spatialisation de l'altérité qu'une société moderne localise en son sein, créant des lieux réservés destinés à protéger ou à exclure, comme les églises ou les prisons, servant d'espaces d'illusion dans lesquels jouer avec ses fantasmes - comme les maisons closes -, ou de compensation où exercer sa capacité à façonner la réalité à son image. C'est dans ce dernier aspect,

selon Foucault, que l'hétérotopie rejoint l'objet même de la colonie. Comme hétérotopie, la colonie vise, en effet, la création d'un autre espace réel aussi parfait, aussi méticuleux, aussi arrangé que le nôtre est désordonné, mal agencé et brouillon ${ }^{7 ”}$. Cette volonté d'ordonner le monde de l'autre à sa propre image est bien au cœur du projet colonial.

Pour y parvenir, les produits de la culture dominante sont utilisés en fonction de leur pouvoir de séduction. Ici, un second mécanisme hétérotopique apparait. Il concerne cette fois non plus le territoire colonisé, mais le territoire symbolique de la métropole coloniale. Les lumières de Paris surgissent de cette hétérotopie d'illusion. Elles nourrissent en cela l'autre versant de la relation culturelle que la métropole exerce sur ses colonies : un exotisme à rebours.

Le vecteur de cet imaginaire doublement colonisé, le bateau dans la cale duquel Kocoumbo trépigne, fonctionne également sur un mode hétérotopique. Le bateau est, selon Foucault, "le plus grand instrument économique" du développement de la civilisation occidentale et aussi sa "plus grande réserve d'imagination". Ce "morceau flottant d'espace" est une hétérotopie "qui, de quartier à filles en quartier à filles, de bordée en bordée, va jusqu'aux colonies chercher ce qu'elles recèlent de plus précieux en ces jardins ${ }^{9 "}$. Voici qu'apparaît, par le bateau, un lien inédit entre les colonies

Durant la période coloniale, le voyage vers Paris est vécu, par tout son cercle social, comme une onction pour celui qui a la chance de partir. et les maisons closes, comme si les parcours d'étudiants qui nous intéressent avaient pour destination inavouée de garnir les galeries d'un bordel à l'échelle d'une ville où la civilisation coloniale aurait le loisir de contempler les créatures de l'imaginaire ranci qu'elle exporte aux colonies.

Plantant l'histoire de son héros, Raymond Spartacus Kassoumi, dans les années 1920, Le Devoir de violence de Yambo Ouologuem, qui remporta le prix Renaudot en 1968, traduit amèrement cette nouvelle modalité de la traite, celle de 
l'élite colonisée : "Les jours que vécut dès lors Raymond furent ceux de toute sa génération - la première des cadres africains, tenue par la notabilité dans une prostitution dorée - marchandise rare, sombre génie manceuvré en coulisse et jeté au-devant des tempêtes de la politique coloniale au milieu de l'odeur chaude des fêtes, des compromis (DV, p. 207)."

\section{Un triangle des Bermudes}

Dans Mirage de Paris, Sidia, fraîchement arrivé, débute sa visite par les Champs-Élysées, la Bastille, la Sorbonne "qui contenait tant de savoirs (MDP, p. 31)", en somme, les hauts lieux de la capitale que n'importe quel touriste s'épuiserait à tenter de visiter en un jour. Dans le taxi, "regardant à travers les vitres les multitudes de maisons défiler, hautes et grises, portes et

D'un quartier à l'autre, la ville éprouve les préjugés du colonisé et laisse entrevoir,

par-dessous sa robe de lumière, les coutures de ses territoires et le tracé de ses sentes de perdition. fenêtres innombrables, toujours closes", il prend vite conscience que Paris est "plus qu'une ville, un monde sans limites précises (MDP, p. 29)". Dans la grande ville qui domine le primo-arrivant de toute l'étendue de sa superbe, les limites sont pourtant aussi ténues qu'infranchissables. D'un quartier à l'autre, la ville éprouve les préjugés du colonisé et laisse entrevoir, par-dessous sa robe de lumière, les coutures de ses territoires et le tracé de ses sentes de perdition. Le Paris des cartes postales et des catalogues n'a rien à voir avec ce que Sidia découvre devant lui : "Il s'était figuré qu'à Paris, du matin au soir, tout le monde était en smoking, occupé à s'amuser. Dans sa construction de la ville, il n'avait pas fait entrer les millions de travailleurs de toute classe qui y vivaient (MDP, p. 32)." La prise de conscience de l'existence de classes laborieuses au sein de la société du colonisateur précède de peu leur rencontre par l'étudiant colonisé, pris dans les affres de la précarité matérielle et du déclassement social. Après moult péripéties, échoué dans un tau- dis du quartier de la Bourse, rue Vivienne, sur son lit rouillé qui l'empêche de dormir, Kocoumbo ressasse sa faim et son amertume: "Pouvait-il prévoir qu'un jour Paris lui offrirait un tel grabat! (...) On n'a pas idée de ça! C'est horrible, tout de même! Avoir quitté sa case pour ce comble infect, ses parents pour une concierge au visage de harpie, ses jeux et sa gaieté pour la famine et le désespoir... (KO, p. 216)."

\section{Vertiges du déclassement social}

Comme Sidia ou Samba Diallo, et à vrai dire comme tous les autres héros des romans étudiés, Kocoumbo vient d'entrer dans cette zone indécise de turbulences traversée par des tourbillons de désillusion, d'impuissance et de lucidité, qui échappe à tout contrôle et conduit souvent à la mort. À l'instar de Sidia, Kocoumbo, dégoûté, projette son rapatriement par le ministère des Colonies. La seule issue aux tourments de la ville relève du colonisateur. Chez Loba et Socé, l'horizon des possibles est encore dans les mains du système colonial. Trois jours avant sa convocation au ministère, Sidia, accablé de tristesse suite à la mort en couches de Jacqueline, une jeune Française qu'il venait d'épouser, se jette d'un pont "dans l'eau froide de la Seine, réchauffée et enchantée par les visions qu'il étreignait dans ses bras (MDP, p. 187)". Kocoumbo, lui, est décidé à ne plus repartir. Avec l'argent du ministère, il rembourse sa concierge et quitte sa chambre. Glissant sur la pente vertigineuse du déclassement social, il ne devra son salut qu'à l'aide d'un administrateur colonial ami de son père. Entre-temps, il expérimente un nouveau rôle dévolu à létudiant noir déclassé condamné à survivre : batteur de jazz à Pigalle, un des territoires ambigus de l'exotisme colonial. "Sa nouvelle situation de batteur le comblait de dégoût. Avoir échoué là, dans ce sous-sol aux lumières tamisées, dans cet orchestre de jazz, le remplissait de rancoeur envers la vie et envers lui-même. (...) Il ne songeait même plus à ses études (KO, p. 251)." 
Pour Fatoman, dans Dramouss, l'humiliation prend la forme d'un travail nocturne dans le ventre de Paris, aux Halles: "Il rencontrait là tous les gars que la vie avait aigris et qui, pour ce motif, avaient un langage peu choisi (DRA, p. 91)." Sans argent à Paris, "la vie devient aussi pénible, aussi solitaire et hostile que dans un désert (DRA, p. 91)". C'est cela les mirages de la ville : les leurres que l'on ne peut s'offrir, posséder pour s'y engloutir, pour y étourdir son esprit, car, si dure et violente qu'il soit pour les uns, Paris n'en demeure pas moins, pour d'autres, une fête.

\section{Le royaume de l'artifice}

Si le dénuement matériel est l'un des écueils de la ville, provoquant la stupeur des héros, il est une cause plus profonde à leur déréliction. Paris ne laisse apparaître son hostilité structurelle aux nouveaux venus qu'après avoir enlevé un à un ses masques et laissé couler son fard. Sidia avait entrevu les syncopes de la vie nocturne scandée par le rythme des dancings. Au retour de l'un d'eux, il constate: "Les danseurs aux épaules larges d'athlète nétaient pas si solides. Le garçon du vestiaire nétait pas aussi aimable qu'il en faisait montre. (...) Il eut de l'Europe l'impression de quelque chose d'artificiel (MPD, p. 47)." Nul n'aura mieux saisi la profondeur abyssale de cet artifice que Cheikh Hamidou Kane, par la voix de Samba Diallo, faisant écho à celle de Rainer Maria Rilke dans les Carnets de Malte Laurids Brigge. Samba Diallo, étudiant en philosophie tourmenté, s'identifie, lors d'une promenade, au personnage de Rilke : "Oui... je suis Malte Laurids Brigge. Comme lui, je descends le boulevard Saint-Michel. Il n'y a rien... que moi... que mon corps, veux-je dire. (...) Il n'y a rien, que mon gros orteil droit. Sinon, leur rue est vide, leur temps encombré, leur âme ensablée là-dessous, sous mon gros orteil droit et sous les événements et sous les objets de chair et les objets de fer... les objets de chair et... (AA, p. 140)." Rilke, qui y a séjourné au début du XXe siècle, fait de Paris l'un des décors de son unique

roman. Comme Samba Diallo, sur le même boulevard, Malte est frappé par la foule besogneuse, ce flot de petites monades indifférentes les unes à l'égard des autres ${ }^{10}$. Dans une lettre à Lou Andréa Salomé, le poète fait part de son saisissement : "Et tous ceux pour qui chaque jour est une souffrance, tous ceux qui dans le tumulte n'entendent pas leur volonté, tous ceux que submerge l'angoisse - pourquoi n'ont-ils personne dans les grandes villes ${ }^{11}$ ?" Pour certains, dit Rilke, Paris est "une forêt perfide, une forêt sans fin 12", et les personnages étudiés sont de ceux-là.

\section{L'épreuve de la nausée}

L'angoisse de Samba Diallo paraît très proche de celle qu'éprouve le personnage principal de La Nausée de Jean-Paul Sartre. Chez le philosophe français, c'est "lexistence nue ${ }^{13}$ " des êtres et des choses qui sature les perceptions d'Antoine Roquentin. Le narrateur sartrien est subjugué par la conscience de "ce gros être absurde ${ }^{14 "}$ qu'est le monde: "Des objets en bois qui ressemblaient à des chaises, à des sabots, d'autres objets qui ressemblaient à des plantes ${ }^{15}$." Sa nausée est le fruit de cette évidence brutale, sans équivoque : le fond de l'existence n'est qu'un "affalement gélatineux", "une ignoble marmelade $^{16 "}$. C'est cette même vision de l'inertie du monde, de "la pâte même des choses" ${ }^{17 "}$ dans le langage sartrien, qui frappe Samba Diallo durant sa déambulation sur le boulevard Saint-Michel. Qu'ils soient de fer ou de chair, seuls surgissent devant ses

La maîtrise consommée du réel, la transformation de chaque facette de la vie en objet de commerce aurait achevé de briser tout lien privilégié avec autrui et le monde. Et la nausée en serait le produit existentiel singulier yeux des objets silencieux. Or, pour Samba Diallo, c'est ce silence du monde qui rompt la possibilité même d'un dialogue existentiel, pourtant essentiel au fervent croyant qu'il est encore, ou plutôt qu'il tente de demeurer. Au comble de la déréliction, il 
s'exclame : "Je suis comme un balafon crevé, un instrument de musique mort. J'ai l'impression que plus rien ne me touche (AA, p. 163)." La prédominance du langage de l'objet chez Sartre et Kane semble renvoyer au cœur même de la civilisation technique moderne. La maîtrise consommée du réel, la transformation de chaque facette de la vie en objet de commerce aurait achevé de briser tout lien privilégié avec autrui et le monde. Et la nausée en serait le produit existentiel singulier.

L'impasse existentielle dans laquelle se trouve Samba Diallo aurait également une portée sociale. C'est ce que suggère Aké Loba. Déçus par la réalité de leurs conditions de vie à Paris, Kocoumbo et son ami Mou ont pris conscience que personne ne les y attendait et, qu'en retour, il n'y avait rien à en attendre. Leur constat est sans appel. Tandis que Kocoumbo s'interroge encore sur les raisons de sa présence à Paris, lui qui se vivait au départ comme un pionnier en mission, Mou lui fait part de sa résignation: "- Qu'est-ce que nous faisons ici ? (...) Je te réponds : rien. Nous regardons, nous attendons (KO, p. 211)." D'autres étudiants ne baissent pourtant pas les bras. Prenant leur part à l'économie de la débrouille, ils rejoignent ces marges de la société occidentale où se débat le lumpen prolétariat. D’après Ousmane Socé, les profils sont variés : il y a les travailleurs précaires, "certains sétaient improvisés musiciens, plongeurs, danseurs dans les boîtes de nuit. D'autres exerçaient à Montmartre un métier rémunérateur et de tout repos. Ils étaient les 'associés' de pauvres filles acculées à la prostitution. Ceuxci ne paraissaient plus dans le milieu des frères restés sérieux (MPD, p. 181)”.

\section{Le bas-ventre de Paris}

Si les "frères restés sérieux" demeurent, tels des Sisyphe modernes, arrimés à leur vie de labeur, les individus sans scrupules s'adonnent au commerce des corps. Les étudiants colonisés font aussi partie de la clientèle qui s'ébroue le samedi soir sous les enseignes lumineuses des rues de Pigalle. Dans Le Devoir de violence, Kassoumi, le seul Noir de sa bande, est d'autant plus hésitant devant cet "essaim de filles, étalant, sous quelque vague habit criard, de lourds seins roses, toutes plantées sur des cuisses de différents calibres (DV, p. 212)", que les "doudou" fusent sur son passage, marques faussement affectueuses d'un exotisme crasse venu des colonies. C'est aussi le cas pour Kanaan Niane, à Barbès.

Le héros de Chaîne, l'unique roman de Saïdou Bokoum, sélectionné dans la dernière liste du prix Goncourt en 1974, traite à fond le thème de la misère sexuelle du migrant à Paris à la fin des années 1960. Kanaan est pétri de traumatismes qui se polarisent dans l'onanisme, la fréquentation des maisons closes et bien plus encore. Pour l'heure, c'est au numéro 3 de la rue de la Goutted'Or que cela se passe. Les bars de Pigalle sont loin. Pour les travailleurs migrants en goguette, le sexe bon marché s'étale dans les rues de Barbès, parmi la foule pressée contre les regards des portes des bordels où "les marmites sont parquées en vrac $(\mathrm{CH}$, p. 66)". Surtout des migrantes. "Vénus hottentotes égarées dans ces musées de graisses en colis, de caissons bourrés de joyaux pourris livrés à l'encan aux vautours émigrés ou non, mais toujours en quête de pain de pute ( $\mathrm{CH}$, p. 68)." Dans le quotidien du migrant déclassé, les miroitements de la Ville-lumière s'apparentent aux reflets d'un lampadaire dans une flaque d'urine.

On voit se nouer, dans les recoins de la trame urbaine, le lien hétérotopique évoqué par Michel Foucault entre les maisons closes et les colonies. Prostituées et étudiants descendent des mêmes bateaux, sont les deux figures complémentaires de la traite moderne, celle des corps et des cerveaux. Pire, comme le navire, le corps du migrant tendrait lui-même à devenir une hétérotopie, une prison qu'il porte avec soi, un espace d'altérisation soumis aux désirs qui traînent dans cette cale pourrie où la société dominante cherche à enfouir ses névroses. 
Le traitement de la relation homosexuelle, que l'on retrouve plus fréquemment après les indépendances, achève de renseigner sur l'exotisation du postcolonisé à l'endroit duquel perdurent les stéréotypes. Dans un bar, Fatoman tombe des nues quand il comprend l'objet de la prévenance bizarre dont un vieil homme fait preuve à son égard: "Des pensées obscures m'étaient revenues à l'esprit : je songeais à ce vieillard qui m'avait lutiné tout à l'heure à la Pergola, à ce fou qui mavait tâté la jambe (DRA, p. 83)." Le Noir est une obsession pour certains. Et le choix peut être fait d'assumer cette réalité morbide et, paradoxalement, d'en tirer profit. C'est le cas de Kassoumi face à l'homme d'un certain âge qui le regarde avec insistance. Pour éviter l'esclandre, au comble de la confusion, enfermé dans le reflet déformant de sa condition colonisée - "Lui, un Nègre. Qu'espérer quand on est nègre (DV, p. 228)" -, il accepte de se soumettre aux volontés de l'homme: "Il n'avait pas le choix. On le désirait et il lui fallait suivre. (...) L'étudiant se leva : il avait accepté de se vendre (DV, p. 229)." Entretenu par son amant durant dix-huit mois, ce rôle de gigolo lui permettra d'achever ses études d'architecture.

\section{Dans la moiteur des édicules}

Rien de beau ne naît dans le lit des représentations coloniales. C'est Chaîne qui ira le plus loin dans la mise en scène du jeu fantasmatique séparant de plus en plus le migrant de lui-même. Au début, Kanaan fréquente "les chiottes des cafés douteux" pour s'y délecter de la lecture de "graffitis obscènes": "D'abord, je me contentais de lire pour que je bande, alors je brandissais mon sexe et le masturbais pendait que je relisais (CH, p. 59)." Puis il en vient à écrire lui-même des annonces et à fixer des rendezvous. En lecteur désabusé de La Nausée de Sartre, cherchant à éprouver ce sentiment aigre-doux de l'existence nue du monde, Kanaan finit par le ressentir... sous un arrêt de bus. Sa brève attente est interrompue par l'arrivée de l'homme venu le ren- contrer: "Je l'avais eue ma première petite nausée. Je piquai un cent mètres. Ce soir-là encore je tombai dans un profond sommeil. (...). J'avais fait des tas de rêves, tous oubliés, qui n'avaient laissé que des traces, trois mots : SAINT JEAN PO (....). En les travaillant un peu, jobtins : SEIN, JAMBES, PEAU. (...) Mais j'ai eu du mal à me débarrasser de SAINT JEAN PEAU qui me suivaient partout, à Barbès, Pigalle, Saint-Denis, le jour, la nuit, et surtout pendant mes séances de plaisirs solitaires (CH, p. 61)."

Dans ce Saint Jean Peau qui cristallise les obsessions de Kanaan, on remarquera la consonance possible avec une autre figure dérivée de Sartre, à l'image de "Jean-Sol Partre", l'anagramme de Boris Vian : un "saint Jean-Paul" en divinité de l'écume des nuits sans lune qui n'éclairent plus rien de la destinée de Kanaan. N'ayant pas encore touché le fond de l'(in)existence de son être, il poursuit sa propre annihilation. Par quelques annotations ethnographiques, le narrateur de Chaîne fait des toilettes publiques de la gare Saint-Lazare l'un des hauts lieux de l'exotisme des édicules. Un amour specDans le quotidien du migrant déclassé, les miroitements de la Ville-lumière s'apparentent aux reflets d'un lampadaire dans une flaque d'urine. tral et macabre de pissotières d'où surgissent des mains venues tâter le sexe du migrant : "Mon voisin, un petit vieux - encore! - au visage hâve, aux lèvres revêches et à la peau toute gercée, avait allongé le bras et effleuré de sa main calleuse mon organe. J'étais confus et je pensais qu'il était dingo, le vieux. J'écartai sa main, pissai rapidement et sortis. Je venais juste d'arriver d'Alger (CH, p. 61)." L'exotisme n'est jamais qu'un avatar du racisme. Et les deux sont intimement liés dans la dérive sexuelle de Kanaan.

\section{Le terreau du racisme postcolonial}

Les migrants postcoloniaux, dont la visibilité augmente dans les quartiers populaires, sont la 
cible privilégiée des racistes. Selon un sondage de 1962, un Français sur 5 pense qu'il y a trop de Noirs en France. En 1967, 52 \% des Parisiens ressentent un sentiment désagréable à leur égard ${ }^{18}$. La tolérance paternaliste de la période coloniale s'est muée en rejet pur et simple. Après avoir sympathisé avec "un groupe de jeunes (voyous de banlieue, dit-on souvent) qui avaient l'air sympa $(\mathrm{CH}, \mathrm{p}$. 71)", Kanaan, va faire les frais de ce racisme postcolonial décomplexé à l'encontre des immigrés ex-colonisés. La virée en "Zédoche" s'éternise et tourne mal. Kanaan se retrouve attaché à un arbre en pleine forêt de Fontainebleau. Les jeunes le violent et l'abandonnent dans
En près de quarante ans de traitement littéraire

les voyages des étudiants

colonisés semblent converger vers ce point fragile où les tourments

postcoloniaux

rejoignent la mobilisation sociale des prolétaires une mare de sang. "Lui qui semble être le chef de bande, me lance en enfourchant sa bécane: - C'est pas quion est raciste, mais on veut plus vous voir dans la zone (CH, p. 74)."

La zone est le pendant de la ville, un espace indéfini de bidonvilles et de terrains vagues qui s'étire aux portes de Paris.

Un espace de relégation qui s'avère, lui aussi, hostile aux migrants postcoloniaux, condamnés à demeurer des autres parmi les pauvres, des errants parmi les déshérités, expulsés aux marges de la marge d'une société qui cherche des boucs émissaires à son entrée en crise structurelle. Cette séquence, à l'issue de laquelle Kanaan n'aura plus en tête que l'idée du suicide du haut des murs du Sacré-Cœur qui surplombe Montmartre, mêle les époques et les pays dans une analogie sublime et désespérante avec le Sud américain : "Mais où suis-je? Quelque part à l'entrée de Nashville dans le Tennessee. Deep South (CH, p. 74)." Avec Kanaan, l'aventure du migrant africain (post)colonisé apparaît dans toute la violence de sa trajectoire chaotique qui emprunte les chemins de traverse de l'histoire, ou plutôt les réunit dans une simple rigole de sperme et de sang.

\section{Conclusion}

Que l'on se rassure, Kanaan ne mourra pas! Mieux, devant les lumières d'un foyer de travailleurs en feu, il interrompt sa tentative de suicide et part porter secours à un migrant qui tente de sauver ses affaires. Son geste lui vaut de sérieuses brûlures et une convalescence à l'hôpital. Il va surtout lui permettre de renouer avec la solidarité des travailleurs africains en lutte pour leur reconnaissance au sein de la société française. En près de quarante ans de traitement littéraire, les voyages des étudiants colonisés semblent converger vers ce point fragile où les tourments postcoloniaux rejoignent la mobilisation sociale des prolétaires, marquant l'exigence de la réappropriation d'une histoire dont l'essentiel se passe à couvert, dans les dortoirs des foyers, dans le tumulte métallique des chaînes d'usine, dans le silence des meublés sous le contrôle des marchands de sommeil. Encore des hétérotopies. Entre les flammes, Kanaan distingue ces rangées interminables de lits de fer superposés jusqu'au plafond: "Il y en a tellement qu'à les regarder de plus près, on verrait qu'il s'agit en fait de landaus. Jolis petits landaus. Mignons petits landaus. Une nursery, c'est cela, le nègre a toujours été pomponné, pouponné (CH, p. 94)." Et il suffit de peu pour que la conscience de l'horrible réalité éclate. Chaîne paraît au début de la grève des foyers de travailleurs africains qui va marquer la décennie 1970. Dans l'imaginaire des migrants, les Champs-Élysées, la Bastille, la République laissent place à d'autres noms, ceux des symboles de l'habitat précaire qu'ils dénoncent et dont ils refusent de payer les loyers : Gallieni, Archinard... Saïdou Bokoum emploiedes noms d'administrateurs coloniaux pour désigner l'hébergement des ex-colonisés. Et l'hétéropie dévoile sa mécanique cynique d'exclusion. Une instrumentalisation brutale qui éclate dans la mélopée fredonnée par un travailleur. Un des maillons de Chaîne : "Ze te fabriqué / Ti me fabriqué / Si ti révolté / Alors ze te fabriqué (CH, p. 138)." 